\title{
A New Tool for Surgical Training in Knee Arthroscopy
}

\author{
Giuseppe Megali ${ }^{1}$, Oliver Tonet ${ }^{1}$, Marcello Mazzoni ${ }^{1}$, Paolo Dario ${ }^{1}$, \\ Alberto Vascellari ${ }^{2}$, and Maurilio Marcacci ${ }^{2}$ \\ 1 CRIM, Scuola Superiore Sant'Anna, Pisa, Italy \\ \{g.megali,m.mazzoni, o.tonet,p.dario\}@mail-arts.sssup. it \\ http://www-mitech.sssup.it \\ 2 Biomechanics Lab, Istituti Ortopedici Rizzoli, Bologna, Italy \\ \{M.Marcacci, A.Vascellari\}@biomec.ior.it \\ http://www.ior.it/biomec/
}

\begin{abstract}
This paper presents an educational method for minimally invasive surgery (MIS) and an integrated system to train a priori knowledge and to exercise manual dexterity. The approach is generally suitable for MIS interventions but has been developed specifically for knee arthroscopy. Based on a classification of the knowledge required for performing arthroscopy procedures, the system provides multimedia modules to train and assess anatomical and procedural knowledge and a virtual reality-based simulator for training perceptual-motor skills. The system is currently being experimented for metrics definition and extended incorporating networked database management.
\end{abstract}

\section{Introduction}

Minimally invasive surgery (MIS) has had a great expansion in the last few years. This kind of procedures introduce great advantages for patients, but also severe limitations for surgeons [3. Although the complication of the surgical techniques impose a longer and more difficult training, the technological development provides new tools, such as multimedia and virtual reality, that can contribute to solve educational problems in the surgical domain. Surgical skills involved in MIS, at the current state of knowledge, can be divided into cognitive and perceptual-motor skills. The development of tools capable to train each of them is a big challenge, as is the definition of metrics for objective assessment of individual progress and comparison of skills between different users [1]. The generation of exercises capable to reproduce the complex relationships between cognitive, perceptual-motor, and experiential factors in addition to the experience of assistants and the quality of equipment used is a very hard issue. Bench models [6] and virtual environments [9] can be used as tools for understanding the development of perceptual motor skills and their relationship to higher cognitive abilities and skills in surgery. Multimedia material, based on images and videos illustrating many complex phenomena [8], can play an important role in training cognitive skills, especially in MIS where the surgeon carries out the 
intervention relying mostly on visual feedback. Evaluation methods are a major issue [5 7]: in fact, metrics for evaluating surgical ability is not unequivocal, and even in a non-technological environment, there have been former studies 4 . where parameters such as aptitude test scores, duration of surgical experience or consultant technical skill rating have shown no significant correlation. The things get even more complex in case of surgical simulators [2].

In this paper, after an introduction of our educational approach, we present a training system integrating multimedia and virtual-reality based modules for separate training of surgical skills.

\section{Educational Strategy}

Our approach to solve the educational problem is aimed at the identification of the educational objectives, followed by the definition of the suitable methods. Our work has been structured aiming at three main objectives:

1. to conceive and formalize a novel educational route for MIS surgeons;

2. to identify the elementary cognitive and perceptual-motor skills to train, and to develop a technological suite, consisting of an organic set of elementary modules, for training and assessment of specific skills;

3. to carry out the experimentation of the method in order to evaluate the teaching effectiveness and to determine the evaluating metrics.

According to our view of the field, the problem of training junior surgeons to a specific MIS technique can be solved in a three-phase process:

1. theoretical study of the surgical technique performed by using interactive multimedia material which has the function of guiding the surgeon through the different steps of the intervention and the understanding of image-based information;

2. training manual dexterity by means of a set of specific exercises focused on the single elementary skills: hand-eye coordination, depth perception, movement synchronization, execution of complex paths, etc.;

3. guidance by an expert surgeon in the execution of the intervention on patients in the operating room.

The presented Knee Arthroscopy Training System (KATS) aims at reducing the learning curve in knee arthroscopy interventions, and at preparing the junior surgeon to his active participation in the operating room. The identification of methods and the definition of specifications for the educational tools has been approached from a general point of view, so that the learning system can be easily ported to other areas of articular surgery.

KATS is divided into three modules:

1. the Anatomical Knowledge Module (AKM) aims at providing a tool for training and assessing the junior surgeon's skills to identify anatomical structures and pathologies, starting from diagnostic images (CT, MRI, $\mathrm{X}$-Ray), direct vision (open surgery, cadaver dissection), and arthroscopic images; 
2. the Procedural Knowledge Module (PKM) aims at providing a tool for training and assessing the junior surgeon's knowledge about the single steps involved in performing a specific arthroscopy intervention;

3. the Navigation Training Module (NTM) consists of a simulator, based on dynamic virtual environments and on a realistic man/machine interface that allows the learner to acquire, improve and assess specific elementary skills needed for moving the surgical tools in a dexterous way during arthroscopic interventions.

\section{Knowledge Assessing and Training Modules}

The AKM and PKM share a common design: both are structured as interactive tests with questions based on interactive image maps (IIM) and multiple-choice questions (MCQ)(Fig. 1). The AKM and PKM provide two running modalities:

1. training: every question has an associated hypertext, explaining the correct answer with text, images and movie clips;

2. test: the aim in this case is to assess knowledge, so during the test phase some parameters are recorded for the evaluation of the performance of the junior surgeon (type and number of the correct answers, difficulty level, time used, repetition of errors).

We classified knowledge in a two-dimensional matrix, where each column represents a different type of arthroscopy procedure (meniscal resection, cruciate ligament reconstruction, chondral fractures debriedment, chondral fractures perforations, synovial plica resection, patellar lateral release) and each row refers to a different type of information which is presented to the learner (external physiological anatomy, external pathological anatomy, image-based physiological knowledge, image-based pathological knowledge, physiological technique, pathological technique). Tests, i.e. set of questions on a specific topic, are generated dynamically, according to parameters like logical sequence, user level, former difficulties encountered, etc. and presented to the learner. The multimedia material is structured in a relational database (implemented in Microsoft Access), comprehensive of theoretical knowledge, medical images and movie clips. Every image and clip has associated MCQs, each with relative answers. Moreover, image maps can be associated to still images: the learner is then required to select a point of the image instead of selecting an answer. The value of the pixel on the associated image map act then as evaluation of the user's response.

\subsection{Anatomical Knowledge Module}

Questions in the AKM are knowledge-oriented. The database contains all the questions, the possible answers and the multimedia material divided by topics. A subset of questions are extracted and presented to the learner, who must demonstrate adequate knowledge of that topic. In the AKM tests, the learner must demonstrate: 


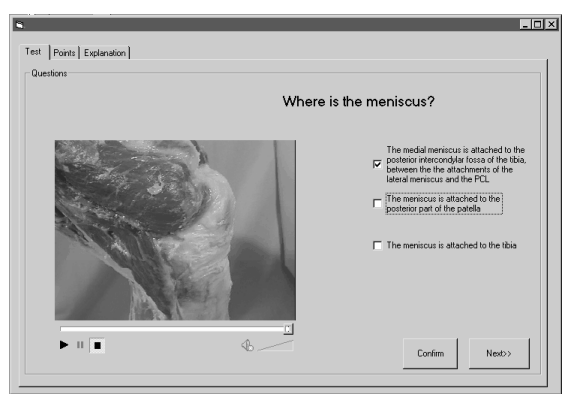

Fig. 1. An example of multiple-choice question.

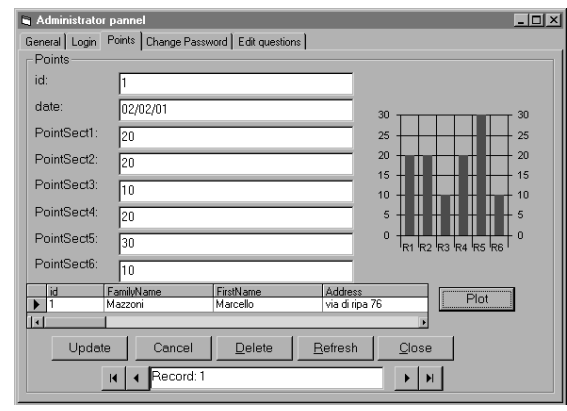

Fig. 2. An example of plot of the learner's performance.

- anatomical knowledge in physiological case, i.e. the ability to correctly identify anatomical landmarks and structures in a variety of cases and image types;

- diagnostic knowledge, i.e. the ability to correctly identify pathologies starting from suitable medical images;

- arthroscopic localization skills, i.e. the ability to recognize anatomical structures in a given arthroscopic image and to determine camera orientation and surroundings.

\subsection{Procedural Knowledge Module}

Questions in the PKM are procedure-oriented, i.e. the learner must demonstrate to possess enough knowledge about a specific surgical procedure to be able to complete all steps required by the procedure, in the correct sequence and being able to take the correct decision in routine and emergency cases. The module is based on movies of specific, selected, surgical interventions, that have been fragmented into elementary, sequential, steps. The multimedia material is created dividing into fragments videotapes of whole arthroscopy procedures of topical cases. Video clips are isolated and catalogued according to the most significant steps. The clips are then associated to questions and presented to the learner in the correct sequential order. The learner must answer the questions and identify the next step of the procedure to be performed.

All the material relative to the single procedures, complete with the associated questions and answers, image maps and evaluation forms are classified in a similar way as in the AKM. The way database queries are made is also the same as in the case of the AKM. The learner must demonstrate:

- step-by-step knowledge of specific surgical procedure, assessed through interactive playback of movies.

\subsection{Evaluation of Performances and Statistics}

In order to have a proper feedback on the progresses of the junior surgeon in AKM and PKM a database of the users is provided. After a learner performed a 


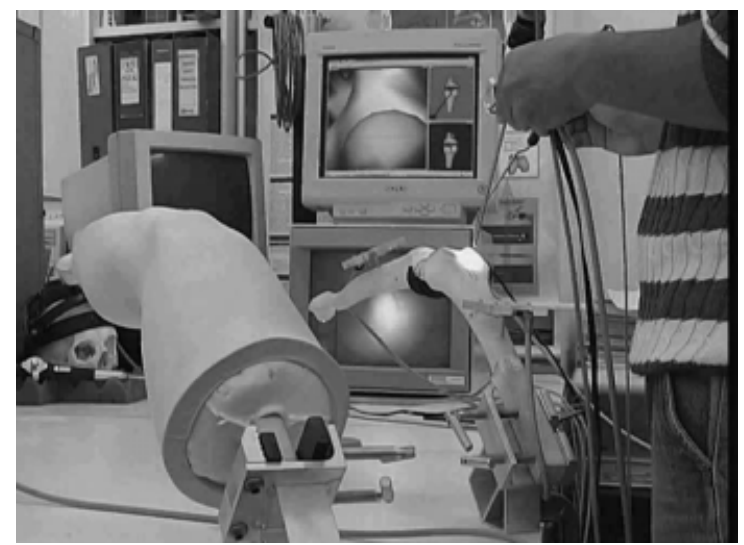

Fig. 3. The NTM module in the experimental setup.

test his performance is recorded in the database, the score is divided by sections and topics according to our classification of knowledge. The results of the tests can be plotted in order to have a visual feedback on the progresses made by the learner over time (see Fig. 2).

\section{Navigation Training Module}

Arthroscopic interventions are performed without directly looking at the site of intervention. The point of view of the endoscopic image varies, according to the arthroscope position and differs in position and orientation from the surgeon's direct vision viewpoint. The perceptual-motor coordination process, necessary to operate in similar circumstances is defined as triangulation.

When performing knee arthroscopy, the patient's leg has to be moved (bent, flexed, stretched) in order to allow or facilitate access to certain knee regions. To keep the leg in the correct position a second operator is needed in the operating room.

The objective of NTM is to train the principal and basic spatial and perceptual motor skills that underlie the execution of a good arthroscopic intervention.

To this purpose we implemented training exercises, in which the learner interacts with dynamical virtual worlds, using real tools for arthroscopy on a mock-up knee. The tools and the mock-up are sensorized to reproduce their spatial position and orientation in the virtual world and to monitor the parameters that contribute to performance assessment.

\subsection{The NTM Setup}

An anatomically realistic knee model, with flexible hip joint and knee joint, is integrated in the NTM so it is possible to reproduce all the position of the knee necessary during the real surgical procedure and to train the second operator (operating under the direct control of the first operator.) 


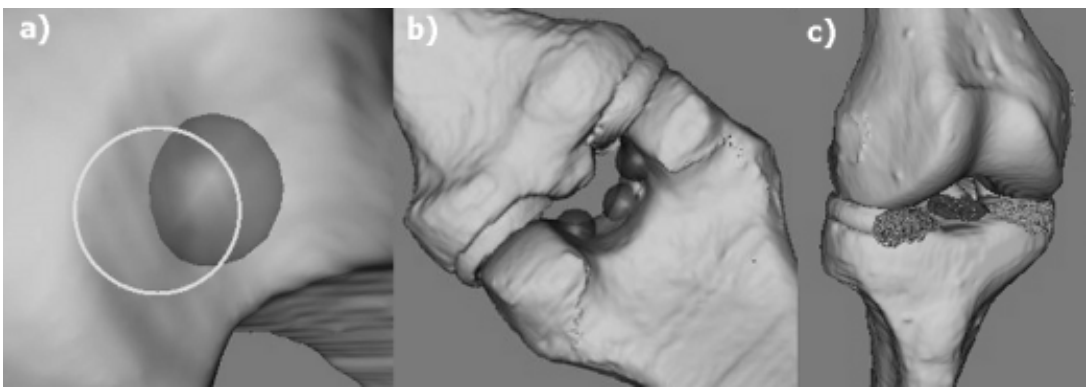

Fig. 4. Virtual Environments for exercises based on colored areas.

The virtual model of the knee has been constructed starting from a CT scan of the mock-up, while the models of the surgical tools have been realized by means of a CAD program.

After the registration step, the movement of the knee mock-up and of the surgical tools are tracked in real-time by the localization system (FlashPoint 5000). For this purpose, frames instrumented with infrared emitters are fixed to femur and tibia, and to the tools.

During the training-phase position information is processed and the virtual environment is opportunely updated and displayed. The data required for performance evaluation are recorded and processed.

\subsection{The Training Exercises}

The virtual environments of NTM combine dynamic representation of the virtual models of the mock-up and surgical tools, with geometrical shapes (or colored areas) located on anatomical landmarks relevant for the training. The exercises created for the training have three basic structures with different training objectives:

Pointing: point to geometrical shapes located on an anatomical landmark of the knee joint with the arthroscope (Fig. 4,a) (single tool management);

Touching: point to geometrical shapes with the arthroscope and touch them with the second tool (Fig. 4.b)(cooperative tool management);

Sweeping: "sweep" or "clean" (i.e. to touch the colored areas with the second surgical tool) some areas in the knee joint (Fig. $4 \mathrm{c}$ )(cooperative tool management and fine movement tuning).

The evaluation of the performance is obtained by measuring time consumption, total path of the surgical tools, number of target misses, and bad contacts with the anatomical parts.

\subsection{The Visual User Interface}

Different types of visual user interface (VUI), designed with different training objectives, are implemented. The VUI based on external and projection views 

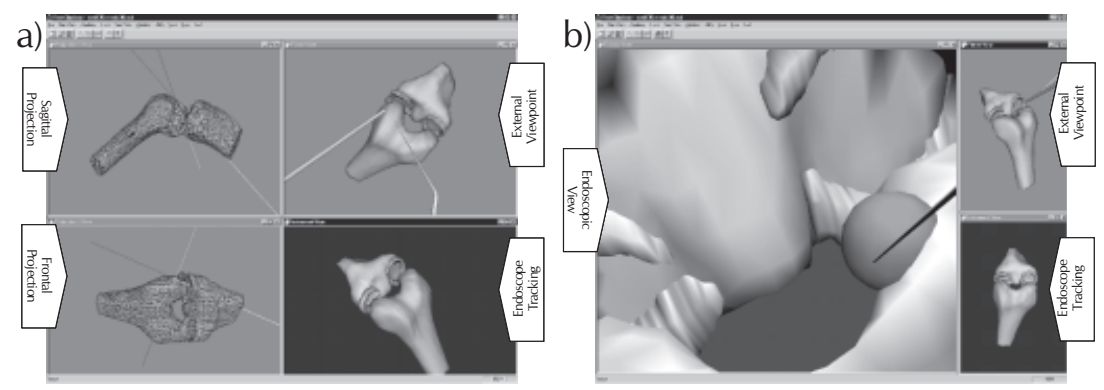

Fig. 5. VUIs for training: global views for training triangulation of the scope and probe (a), endoscope view with assistant views for adaptation to the arthroscopic view(b)

(Fig. 5]a) addresses the training of tool management, whereas the VUI where the virtual endoscope view is dominant (Fig. 5.b) addresses adaptation to the arthroscopic view.

The NTM module runs on a Windows NT 4.0 workstation, Intergraph TDZ 2000 GX1 (Pentium II Xeon 450 MHz, 512 MB RAM, Intense3D RealiZm II). The software has been developed with Microsoft Visual $\mathrm{C}++6.0$, using MFC library for windows management and OpenGL Optimizer for 3D visualization.

\section{$5 \quad$ Preliminary Results and Performance Assessment}

In the development of a training tool, the experimentation phase is important for validating educational effectiveness, and for fine-tuning of the parameters that determine the final metric for evaluation of the learners' performance.

We have preliminary tested the AKM, PKM and NTM in making three surgeons (a junior resident, a senior resident, and a certified arthroscopist) perform a series of tests in the same conditions. The results show that performance (evaluated on the basis of time consumption and number of correct answers) increases with the degree of experience in the surgical room.

To compare inter-operator performance, we should be able to determine apriori the basic surgical skills of a surgeon. The methods of evaluation of the learners' performance and the choice of the parameters to be measured will be refined progressively during the oncoming experimental phase. Several users with different degrees of expertise will test the three modules and reference values for the final version of the formulas will be computed by means of statistical data analysis.

\section{Conclusions and Future Work}

In this paper we presented a training system aimed at providing increased experience of arthroscopy to the junior surgeon. The system, developed in the context of the VOEU 3 Project, consists of modules for separate training of cognitive

\footnotetext{
${ }^{3}$ Virtual Orthopaedic European University (VOEU), \#IST-1999-13079 EU-IST Programme.
} 
and perceptual-motor skills. Preliminary results encourage the prosecution of the research. Future actions will focus on client/server web access for remote generation of tests and management of student profiles, on experimental validation of the system, and on the establishment of suitable metrics for performance evaluation.

\section{References}

1. S. Peterson-Brown A. M. Paisley, P. Baldwin, Feasibility, reliability and validity of a new assessment form for use with basic surgical trainees, The American Journal of Surgery 182 (2001), 24-29.

2. C. Cao, C. MacKenzie, and S. Payandeh, Task and motion analyses in endoscopic surgery, Proceedings of ASME Dynamic Systems and Control Division (K. Danai, ed.), 1996 ASME International Mechanical Engineering Congress and Exposition, 1996, pp. $567-573$.

3. A. Faraz and S. Payandeh, Engineering approaches to mechanical and robotic design for minimally invasive surgeries (MIS), ch. 1, pp. 1-11, Kluver Academic Publisher Group, 2000.

4. A.M. Paisley, P. Baldwin, and S. Paterson-Brown, Relationship between aptitude testing, simulation and technical skill rating in the assessment of surgical trainees, ASME Annual Scientific Meeting, 1999.

5. J. Patrick, Training: Research and practice, Academic Press, San Diego, CA, USA, 1992.

6. D. J. Scott, P. C. Bergen, R. V. Rege, R. Laycock, S. T. Tesfay, R. J. Valentine, D. M. Euhus, D. R. Jeyarajah, W. M. Thompson, and D. B. Jones, Laparoscopic training on bench models: Better and more cost effective than operating room experience?, Journal of American College of Surgeons 191 (2000), no. 3, 272-283.

7. W. Sjoerdsma, Surgeons at work: Time and actions analysis of the laparoscopic surgical process, Ph.D. thesis, Delft University of Technology, Delft, The Netherlands, 1998.

8. B. K. Smith and E. Blankinship, Justifying imagery: Multimedia support for learning through explanation, IBM Systems Journal 39 (2000), no. 3-4, 749-767.

9. F. Tendick, M. Downes, T. Goktekin, M.C. Cavusoglu, D. Feygin, X. Wu, R. Eyal, M. Hegarty, and L.W. Way, A virtual environment testbed for training laparoscopic surgical skills, Presence 9 (2000), no. 3, 236-255. 